\title{
Using Multimedia Case Development as a Critical Reflective Tool for Revitalizing School Stakeholders’ Organizational Learning
}

\author{
Joseph Claudet \\ Department of Educational Psychology and Leadership, Texas Tech University, Lubbock, USA. \\ Email: joe.claudet@ttu.edu \\ Received May 20 ${ }^{\text {th }}$, 2011; revised June $2^{\text {nd }}, 2011$; accepted June $8^{\text {th }}, 2011$.
}

\begin{abstract}
This article reports design and development efforts associated with one school-university collaborative partnership project focused on assisting K-12 school community stakeholders struggling with difficult school improvement challenges learn how to reinvent themselves as organizational teams to engage effectively in positive school change and renewal. The project employs an alternative, immersive organizational learning design in conjunction with available multimedia technology to leverage stakeholders' own lived experiences and leadership challenges as opportunities to engage school community participants in a unique organizational case development and team learning adventure. Multimedia case development and production activities involving school community stakeholders in one elementary school are highlighted. Insights derived from the use of multimedia case development as an organizational leading and learning tool for educators and other stakeholders in school communities are discussed.
\end{abstract}

Keywords: Organizational Learning, Multimedia Case Simulations, Collaborative School Leadership

\section{Introduction}

As an organizational behavior researcher and school improvement consultant serving K-12 schools over the past two decades, I've always been fascinated by the challenge of helping groups of school community stakeholders who find themselves confronting difficult real-world teaching, leading, and learning dilemma situations. And, my fascination is multiplied when the educators and community members I am working with are grappling with dilemma challenges that, over time, have become so organizationally entrenched and intractable that they often require school stakeholders to reinvent themselves as learning communities. This process of reinvention is, by necessity, often systemic in nature and can be destabilizing and fraught with uncertainty, as it demands that school community stakeholders find new ways to refashion their organizational identities and reenergize their capacities for collaborative leadership. More often than not, this collaborative process of "reinventing organizational shared identity and purpose" involves large groups of school community stakeholders learning how to come together to think differently and work together in new ways - in short, to learn how to work together anew as a genuine organizational team.

Learning how to work together as an effective organizational team can be a daunting task, particularly when the dilemma challenges organization members are facing are high-stakes in nature, multi-leveled, and potentially game-changing. The challenges can impact directly the organization's continued effectiveness and stability. Intriguingly, there is a fascinating example of this very kind of "high-stakes" dilemma situation in a suspenseful scene toward the end of Christopher Nolan's mind-bending 2010 movie Inception, in which a team of dream inceptors, hired by a wealthy corporate entrepreneur to plant a new, monopoly-busting "organizational idea" into the mind of a competitor, becomes stuck in a multi-layered, multi-dimensional dream reality of their own creation (a "dream-withina-dream-within-a-dream”). The team finally realizes that the real challenge (and the only way to successfully resolve their predicament and achieve their organizational goals) is to learn how to "tag-team". That is, the inception team members must learn how to work together synergistically and collaboratively as a real organizational team. Team members must find creative ways to genuinely collaborate in practical, meaningful, results-driven ways with other team members operating within the various dream levels through recognizing and valuing each member's core values and beliefs (each member's individual "dream perspective") and how individual members' various "perspective-driven" values and beliefs inevitably inform the group's collective leadership vision. Because, as the inception team learns, if you fail to take into account individual members' core values and beliefs and how these values and beliefs can impact the organization, then team members collectively will not really be "collaborating" (and, in fact, various team members may even be working at cross-purposes with each other). And, as a result, the team overall will not be able to construct a coherent shared leadership vision (a group-constructed vision that successfully integrates multiple individual perspectives) and will not function effectively. In short, the intrepid band of dream inceptors must learn how to "tag team as an organization" (viz., learn how to genuinely think differently and work together in new ways as a functioning team) to be able to successfully implant the new organizational idea, navigate through the multiple dream levels, and "kick" each other back to reality.

Interestingly, the "multiple layers of dreaming" the Inception team members must navigate in within the movie is akin to the "layers of dreaming" (i.e., individual classroom lesson-planning mindset, grade-level unit planning demands, etc.) that teachers 
in schools can sometimes get trapped in. As a result of the longstanding organizational "structures" in place in K-12 school organizations (individual classrooms, grade levels, etc.), teachers in schools can often become so caught up in their own individual classroom teaching and learning environment survival issues (their "individual trees") that they are unable to see the bigger picture (the "whole school-wide curricular and instructional forest"). As a school improvement consultant, the challenge involves finding ways to help these teachers, along with their school community stakeholder colleagues, mentally expand their horizons - to get out of their own individual teaching, leading, and learning "silos" (individual role perspectives) and discover as a group how to connect the school's individual curricular and instructional dots into a unified organizational pattern (to develop a shared school leadership vision as a functioning organizational team). In short, the organizational learning challenge is to help school stakeholders learn how to "tag team" as a leading and learning community (through learning how to think differently and work together in new ways). It is through this process of learning how to think differently and work together in new, creative ways that school community stakeholders confronting tough, real-world dilemma challenges in K-12 schools will be able to develop the collaborative leadership capacity to forge and renew their own shared organizational visions of who we are and where we should be going as cohesive teaching, leading, and learning communities.

This article highlights a group of school community stakeholders in one elementary school who were struggling with a multi-leveled teaching, leading, and learning dilemma situation of their own making, and reports on their collaborative efforts within a multimedia case development project to learn howas an organizational team-to think differently and work together in new ways.

\section{An Elementary School Community in the Midst of an Instructional Leadership Dilemma}

As part of my ongoing school improvement work with a number of schools in the Permian Basin region of West Texas, I was invited in the fall of 2008 by the principal of one K through $6^{\text {th }}$ grade campus in the region to work with the school's teachers, instructional support staff, and other school community stakeholders who were grappling with the difficult challenge of finding a creative way to break out of the school's recurring pattern of low test scores and move their school community forward. I was intrigued by the principal's description of her school's challenges and the roadblocks she felt she and her colleagues were confronting, and I agreed to meet the school's teachers and support staff and learn more about their situation.

After spending some time getting to know the teachers and their school's instructional setting, I began to sit in on various academic team planning meetings involving grade-level faculty. As I sat and listened to teachers during their weekly planning meetings, one striking feature I observed that was reflected in teachers' discussions within and across different grade-level teams was the evident passion that teachers brought to their instructional work and their resounding commitment to their students. Within one fourth grade team, for example, the teachers involved spent a considerable amount of their planning time (over a period of several weeks) discussing the "instructional problems" they were experiencing as a result of their students' evident technological savviness in being able to access and download information about dinosaurs from the worldwide web. As one teacher lamented, "my students are naturally attuned to the wealth of information resources available on the internetI guess it's just a natural part of their social heritage in this new, interconnected age. They are already very adept at searching for and downloading information from the net, and they are constantly challenging me to keep up with them.” Several other teachers in this fourth grade team nodded in agreement. As I continued to observe these teachers during their planning meetings, it became clear that one overall perception residing in these teachers' minds (reflected in that one teacher's frank comments) was that these teachers felt that many of the students in their classes were far ahead of the overall "instructional pace" that the teachers had designed and were attempting to employ within classroom activities as part of their fourth grade "dinosaur unit". And, this created a growing sense among the teachers that, rather than working proactively to "instructionally plan ahead" for their classroom teaching, they were spending much more of their planning time just trying reactively to "keep up with their students".

Other grade-level teams I visited seemed to echo, in similar ways, the fourth grade team's general instructional concerns and reactive strategies within their own planning efforts. One sixth grade team of teachers, for instance, was intently focused on working to find a funding source and write a grant that would support an intensive redesign of their sixth grade social studies instructional units. In yet another team, fifth grade teachers were spending a good deal of their planning time brainstorming ways to actually limit the extent to which their students could utilize "online learning information resources" in completing their written lesson assignments. These fifth grade teachers felt strongly that these online resources were becoming a "crutch" that their students were relying on too heavily_inhibiting students' own creative thinking and writing development.

As I continued to sit in on various grade-level teams of teachers during their weekly planning meetings, a similar pattern of teacher thinking cutting across the teams began to emerge. The various grade-level teams of teachers at this elementary school were fixated, to a large extent, on their own individual and predominantly reactive approach to curricular design and instructional delivery, and were focused singlemindedly on grappling with their own perceived classroom (i.e., grade-level) instructional issues and challenges without really considering any broader (i.e., school-wide) teaching and learning picture.

In my ongoing conversations with the principal, the principal readily admitted up-front that she was not sure how to address what she believed to be some of her school's most intractable challenges - the recurring pattern of low test scores and teachers' entrenched instructional attitudes and beliefs. To inform our discussions, I shared with the principal my own observations regarding what I felt were the somewhat small-lens, reactive instructional strategies many of the school's grade- level teams of teachers were employing within their grade-level classroom planning. As our conversations progressed over the span of a few weeks, and as the principal's own reflective thinking continued to evolve regarding the scope of her 
school's dilemma challenges, the principal began to articulate her evolving suspicion that her teachers' well-intentioned but disjointed and fractured approach to instructional planning might, in fact, be contributing to (and even exacerbating) the recurring cycle at this school of poor teaching and learning results (low test scores) and growing parental complaints-the very dilemma challenges that were fueling the superintendent's insistent demands for improved academic accountability at this elementary school. The principal admitted that she and her teachers "are already implementing everything we can think of to turn our school around, but the results we all want are just not occurring”. Based on my accumulated observational data of teachers and other instructional staff at this school, I suggested to the principal that what she and her teachers needed was to approach their school community challenges in a fundamentally different way-not from the vantage point of individual role players or even grade-level instructional units, but as a school community-wide organizational team.

Because of my related work over the past decade with several other school communities in the region who were also grappling with the ongoing challenge of identifying and implementing their own organizational visions of academic planning and instructional coherence, I was very familiar with the power of organizational futuring as a useful tool for assisting communities of diverse school stakeholders wrestling with entrenched dilemma challenges and confronting the need for systemic change. One of the best staff development applications of the organizational futuring concept I have used over the years has involved conducting a series of future search conferences with multiple role players within a school community. Future search conferences (Weisbord, 1992) have been used extensively in business organizations as a means for organization stakeholders to come together to brainstorm collaboratively in order to construct a shared sense of their organization's history, its overall trajectory, and desired future. As such, future search conferences can be a very useful technique for bringing together "fifty to seventy people, drawn from all parts of the organization and from external constituent groups, [to] work intensely together to create shared visions of the organization's past, present, and future; the whole system is in the room, generating information, thinking about itself and what it wants to be" (Wheatley, 1992: p. 66). The future search conference format becomes a unique opportunity for participants to share member stories and perspectives on the organization's past, to assess the organization's overall condition in the present environment, and to leverage these multiple perspectives and assessments to envision future scenarios, identify common ground, and develop collaborative action plans to achieve the kind of optimal organizational future members want. I have found this organizational conferencing technique particularly useful in situations where the challenge for the external consultant is to help organization stakeholders (large groups of educational and community leaders in K-12 school teaching and learning communities) come together to build or revitalize their collective organizational identity and collaborative leadership capacity.

With some coaxing (and a little persistence), I was able to convince the school principal-and, importantly, the school's expanded campus improvement team (consisting of teacher representatives from each of the school's grade-level teams, as well as a number of instructional support staff, parents, and community members) - to engage with me in January and February of 2009 in a series of future search conferences as an opportunity for the principal, teachers, parents, and other interested school stakeholders to come together and explore their school community's recent history and envision its future.

As the future search conferences commenced, I emphasized to school community participants that the conversations that take place in the future search conference meetings would be challenging (and even unsettling), but the most positive results would occur if participants were willing to share with each other — with as much openness and honesty as possible - their individual perspectives on the challenges facing the school. It didn't take long for individuals participating in the conference meetings to start opening up and sharing their views regarding their school's challenges. Several of the same teachers who had raised concerns during their grade-level team planning meetings about the challenges of dealing with the widespread availability of internet-related resources and their questionable impact on the quality of students' learning voiced their perspectives during the future search meetings. These teachers were quite vocal in complaining about the added challenges to their classroom instructional planning that they felt the proliferation of online resources and communication tools had engendered. Furthermore, these teachers expressed their belief that the challenges of navigating and dealing with these new technology resources were compounding the difficulties they were already facing in developing and delivering quality instruction in their classrooms.

After listening to several teachers share their perspectives, some of the parents in the conference meeting became fidgety and began to share questioning glances with each other. One parent who looked especially uneasy, eventually stood up, turned to face the group, and stated that she'd like to share her own, albeit somewhat different, perspective: “I'm speaking as a parent, not a teacher. But, I currently have three children attending this school in different grades. My children are all growing up in an internet-connected world, and I want them to be able to reap the benefits of technology to help them be successful in their lives. So, I don't understand why teachers are complaining about the internet and how it's disrupting their teaching. I want my kids to learn with the internet tools that are available, and I want their learning to be enriched through participating in well-designed lessons that include web learning activities. Rather than the teachers complaining about technology, I think it's high time the teachers in this school embrace technology and learn how to work together to make technology a vital part of students' learning!” Several other parents in the conference room nodded in agreement. This proved to be an emotional tipping point in the series of search conference meetings, one that, as an organizational learning consultant, I seized upon to introduce to the school community members present an intriguing idea that perhaps they had not yet considered: the idea of actually using some of the very digital technologies that teachers were complaining about in a new, creative way-as an organizational case development tool that school improvement team members could employ together to study and learn from their own instructional dilemma situation.

Further group discussion served to engender a heightened sense among participants of the pressing need in their school 
community for positive organizational change, and at the final search conference meeting the members of the expanded campus improvement team admitted that they had exhausted their ideas on how to address their school's effectiveness challenges, and that it was time to consider new, "outside-the-box" options. Thus, with a sense of adventure (and not a little trepidation), campus improvement team members along with the principal agreed to take me up on my challenge to participate as a team in an alternative staff development project for the coming school year: to use multimedia technology and work together in a new way to develop an organizational learning case about their own school community instructional dilemma.

\section{A School Community Embarks on an Organizational Team Learning Adventure}

Organizational case development efforts implemented over a two-year period at this elementary school campus in the West Texas Permian Basin were grounded in and part of a decade-long multimedia case simulation research and development project supported through initial funding in 1996 through 1998 (totaling US \$400,000) provided by the Sid W. Richardson Foundation (Fort Worth, Texas), the Abell-Hanger Foundation (Midland, Texas), and the Franklin Charitable Trusts (Post, Texas). The initial funding supported the creation of a multimedia case simulation research and development lab in the Texas Tech University College of Education. This multimedia R\&D lab was equipped with broadcast-quality betacam SP cameras, audio microphone and sound mixing equipment, and digital nonlinear editing system hardware and software to enable teams of university project investigators to identify and work with school stakeholders in a number of regional schools and school districts across the West Texas and Texas Panhandle regions who were grappling with a variety of interrelated challenges dealing with school improvement and instructional effectiveness. A central feature of this school-university partnership endeavor involved university-based R\&D personnel collaborating with teams of school community stakeholders in participating schools and school districts to develop organizational learning cases focusing on difficult, real-world school community challenges experienced first-hand by the educators and community members in these K-12 school community environments.

Project case development work at this West Texas elementary school began in earnest during the spring of 2009 with the school's expanded campus improvement team (consisting of teacher representatives from each of the school's grade-level teams, along with a number of school instructional support staff, parents, and community members-all of whom were familiar with and fully involved in this school community's current dilemma challenges as real-life stakeholder participants) comprising the school-based case development team. This schoolbased team began working collaboratively with the university case project team (comprised of film crew audio and video recording personnel, digital non-linear editing and post-production specialists, and elementary school leadership consultants from the regional education service center) who were assigned to this school to facilitate organizational case development and to handle the technical aspects of case filming and post-production work. These teams joined together in a unique school-university partnering endeavor as a multimedia case production team to develop an organizational case focusing on the unique teaching, leading, and learning dilemma challenges of this elementary school community.

Initial project activities involved assisting school-based team stakeholders as they engaged in the process of storyboarding and drafting of individual case scenes that would portray important interactive events, circumstances, and issues relating to the overall case dilemma situation. This preliminary story boarding and scene scripting work was a very important part of the overall case development process, as it directly informed stakeholders' organizational learning. These initial case development activities required school stakeholders to spend time specifically discussing and reflecting on the history of issues, interactions, and events at their school (including multiple and often conflicting stakeholder perspectives on how to appropriately address the school's overall instructional improvement and effectiveness challenges) that, over time, had accumulated and were combining to form the contours of the school community's present dilemma situation. School-based team members worked diligently over a period of several weeks to develop an accurate storyboard depiction of the history of their case situation, along with carefully constructed scripted case "scenes" that depicted representative multiple interactions among various role players/stakeholders, highlighting their often-conflicting perspectives on important school improvement issues.

One important organizational learning feature built into the case development project design involved the fact that, for the filming of individual case scenes, individual school community stakeholders who were members of the school-based case development team were required to take on and "enact" different stakeholder roles than the ones they played in real life. This meant that all school-based participants involved in acting out the various scripted case scenes had to spend a good deal of time learning their new "case roles"-and, in so doing, necessarily reflecting critically on the core beliefs and perspectives of the alternate stakeholder roles they were acting out, rather than simply staying focused on their own real-life role perspective. This "multi-perspectivist” kind of critical examination and reflection by school team participants on case issues and multiple role player values and beliefs was a key organizational learning design element built into the overall multimedia case development process. This level of organizational reflection was specifically designed to assist school stakeholders in learning how to think differently about each other, as well as to develop-both individually and as an organizational learning team-a more nuanced, inclusive view of the multiple factors and perspectives influencing their overall school community dilemma situation.

University-based project team members observed an interesting phenomenon develop and intensify as they continued to work with school-based stakeholder team members during the overall case development process. Even during the preliminary process of storyboarding and script writing in preparation for filming of case scenes, individual school-based team members were keen on making sure their own individual and/or group perspectives (as teachers, parents, grade-level team members, etc.) on important case issues were accurately represented in scripted dialogue that would become part of multimedia case scenes. As school-based stakeholder teams and project film 
crews began in earnest on shooting case scenes, this tendency by school stakeholders to insist on the accuracy with which "their own" real-life role perspectives were portrayed became even more pronounced. It became a frequent occurrence during scene filming for one or more school team members to call for a "timeout" from the shoot. "Wait a minute", one role player would exclaim, and then proceed to challenge another role player: "You're not articulating my perspective [on an important case-related issue] in the right way-you're not conveying convincingly how I really think and feel about this issue.” Additionally, individual case actors were often heard by project film crews during breaks in filming "coaching" their school team colleagues (who were enacting their real-life roles) on the nuances of their own specific role perspectives, and how to accurately inflect and portray their strongly held values and perspectives on case issues through the scripted scene dialogue. The importance of these break-out coaching sessions as a meta-analytic organizational learning dimension of case development activities was recognized early on, and several of these informal multiple team member "coaching sessions" were filmed and later incorporated as a component of the final multimedia case.

Some key features included within the overall multimedia case simulation design template are illustrated in Figures 1, 2, and 3 . The primary multimedia case environment (Figure 1) is designed as a "school leadership office" interactive interface that enables users to access multiple kinds of school-related information databases (e.g., student demographics, multi-year school performance and accountability data, school district policies, state and national school leadership performance standards, etc.). Case users (the school's case development team stakeholders themselves, as well as other educators who may want to use the case to inform their own school-specific organizational learning) can navigate this multimedia environment to: 1) access and review case video scenes; 2) obtain information regarding specific school leadership national and state standards relevant to the case; 3) interact with online mentors (e.g., regional education service center curriculum and instruction consultants, state education agency personnel, uni-

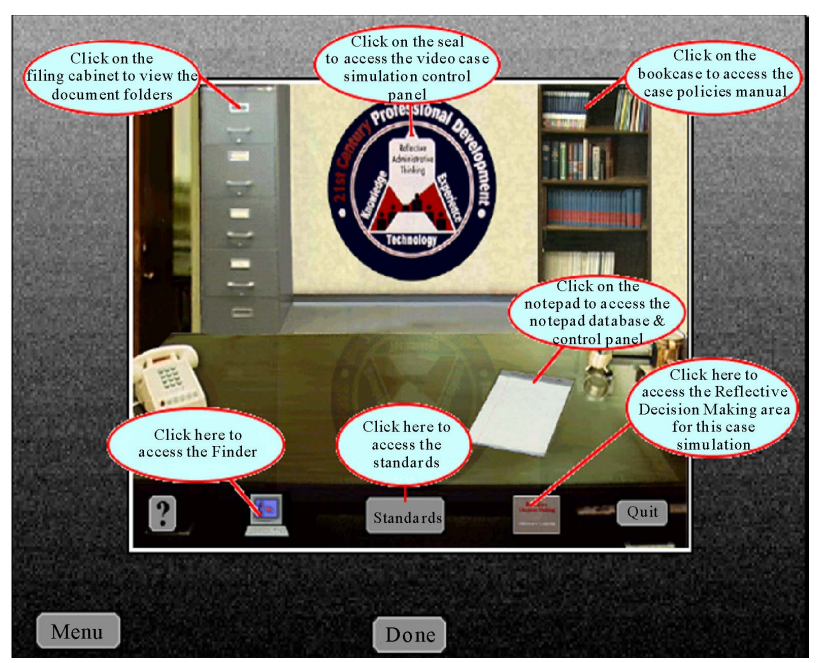

Figure 1.

Multimedia case "school leadership office" interface and interactive environment.

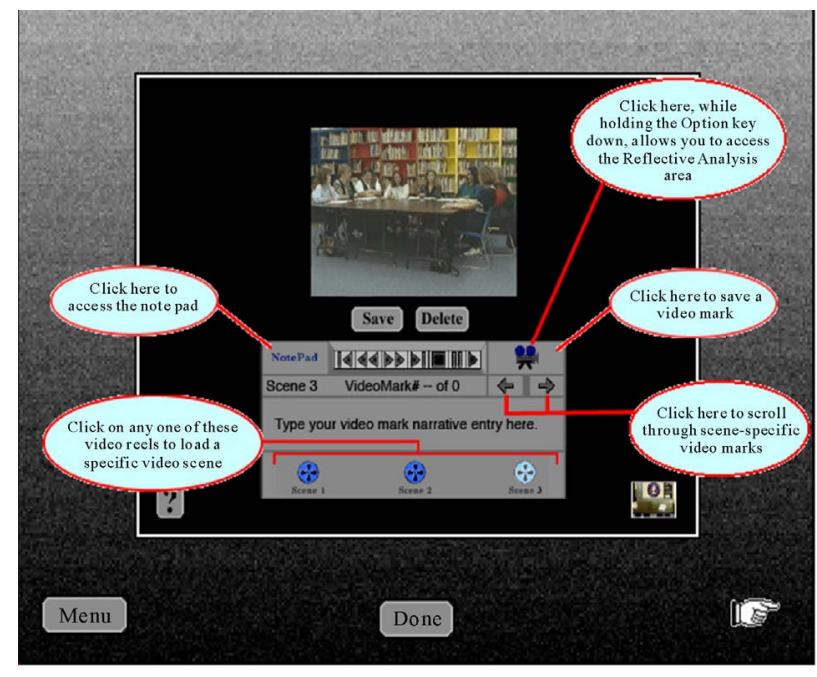

Figure 2.

Case video scenes database with "video mark" functionality.

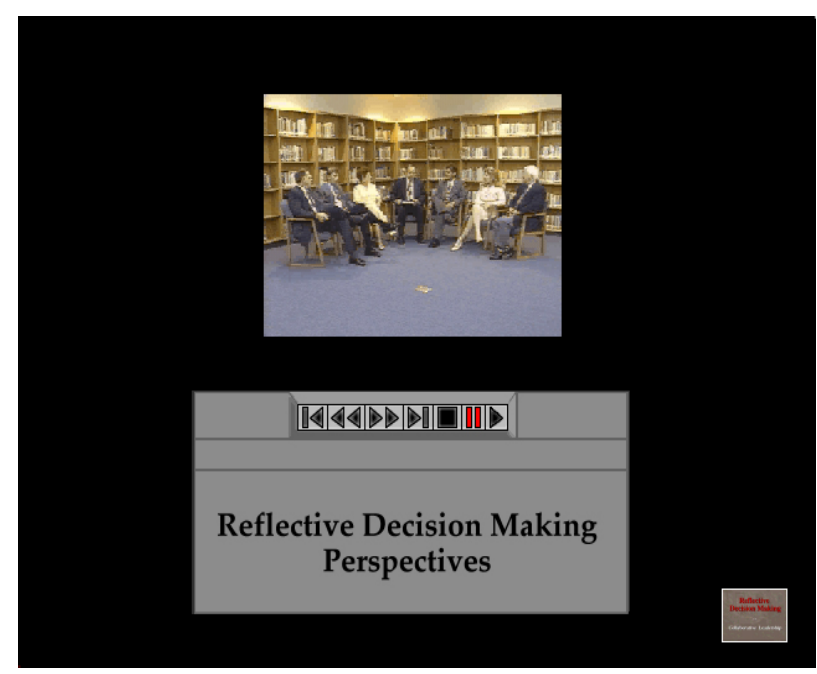

Figure 3.

Expert panel video sequences presenting various case perspectives.

versity professors); 4) search case-relevant information databases contained in digital file folders included in the multimedia environment; 5) develop (both individually and in groups) their own critical reflective analyses of case scene portrayals which can be archived digitally for future sharing with school stakeholder team colleagues; and 6) work together as a collaborative team to brainstorm and develop case-specific school improvement action plans.

Users can access the case's video database of interactive scenes portraying critical incidents relating to the school's case dilemma situation through the Case Video Scenes Database (Figure 2). This database includes a special "video mark" function capability that enables users to digitally mark specific sections of video for further analysis (Figure 2). Utilizing the "video marking" tool, users can frame and analyze individually selected sections of the various case video scenes, and hyperlink their selected scene clip analyses to relevant information available in other video, graphic, and text databases included 
within the multimedia case environment (e.g., school community demographics; grade- and school-level student performance information; expert panel perspectives; etc.). Working individually or in groups, school stakeholders navigating within the multimedia case environment can utilize their selected video clips to carefully examine specific interactive elements of the multi-stakeholder case scene portrayals and develop reflective narrative analyses which can then be directly entered and stored digitally in the case's organizational learning program.

The simulation's Case Reflective Decision Making Area provides an opportunity for case users to directly apply organizational leadership insights about the case dilemma situation gleaned from their scene clip (video mark) individual and stakeholder group reflective analyses to develop case-specific school improvement action plans. This area includes short "expert panel" video sequences featuring the leadership perspectives of a number of seasoned administrators, school community leaders, and educational consultants as they discuss some of the key organizational issues and stakeholder interactive dynamics portrayed in the multimedia case scenes (Figure 3). School community stakeholders can reflect on how these expert panel perspectives might inform their own collaborative leadership thinking as they work to forge and refine their school's leadership team vision and craft their school improvement action plans.

Collectively, the above interactive features incorporated into the overall case simulation design template are intended to stimulate school stakeholders to: 1) critically reflect on the multi-perspectivist dimensions of their school community's organizational dilemma challenges; 2) leverage this critical reflection to develop a more collaborative and integrated leadership team vision of organizational change; and 3) then apply the organizational leadership insights gleaned to formulate some practical decision making action strategies that might result in positive school change and improvement. The final context-specific multimedia case simulation that was developed by the combined project team of elementary school community stakeholders and university-based multimedia specialists reported on in this article became one case installment in an ongoing series of K-12 school leadership case simulations supported by the multi-year project grants focusing on organizational change and instructional improvement.

\section{Discussion}

The collaborative efforts of university-based research teams partnering with school-based teams of school community stakeholders in this and other regional schools in the kind of multimedia case development project reported here have engendered a number of insights among $\mathrm{R} \& \mathrm{D}$ project participants on the potential of multimedia case development as a creative, alternative staff development design and development tool for revitalizing K-12 school stakeholders' organizational learning. Several of the insights derived are discussed below.

\section{Immersion in Organizational Case Development was Seen as a Catalyst for Engendering New Kinds of Multi-Stakeholder Reflective Thinking}

School community stakeholder involvement in the year-long multimedia case development project reported here served as an alternative, immersive organizational learning opportunity for the grade-level teams of teachers, parents and community members, and the school's principal who came together in a new way to explore their school's instructional leadership dilemma challenges. The interactive, multimedia case story this school community case development team constructed enabled team members to explore and critically reflect on their collectively-shared lived experiences in new ways through harnessing the representational power of multimedia technology. A central thrust of this case development project was leveraging available digital story-telling tools to empower school community stakeholders to approach their own professional and organizational learning in new ways. In particular, project activities enabled participants to work together in a new way as a multimedia development team to recreate their lived experiences with the added advantage of being able to critically explore-collaboratively and reflectively in hindsight - the multiple layers of stakeholders' perspectivist beliefs and thinking that were contributing to and infusing the organizational fabric of their dilemma situation.

A number of stakeholder team participants (including several experienced teachers) came to the case development process harboring their own deeply ingrained beliefs regarding what they considered to be the limited possibilities of the internet and related information technologies for creating any real added value to the teaching and learning process. For these participants, teaching and learning was a clear and well-defined process framed by the curriculum and contained within the familiar "bounded" structure of the classroom environment. Other teachers on the case team openly acknowledged the wealth of information content available through the newer internet-enabled technologies, but were less than confident in their own abilities to sort through and leverage these resources in instructionally appropriate ways. Overall, teachers participating in case development activities, in general, were not as yet open to what these new information tools-as well as their own students-might be able to teach them about the evolving nature and possibilities of $21^{\text {st }}$ century classrooms. As "digital immigrants"-individuals who had to make a conscious effort to learn the new digital language of computers and the internetthese teachers were not as comfortable with the internet and information search and communication technologies as their students. Their elementary grade students, by contrast, were all born "digital natives", for whom computers and the internet were a natural part of their digital heritage (Prensky, 2010). Because of this, several teachers during case development team reflective sessions shared their fears about not being able to "keep up with the learning curve in being able to identify and incorporate appropriate internet resources into their instructional planning" and not being able to "stay ahead of their tech-savvy students". As these educators and school community members participated in case development activities, a key part of team members' ongoing group reflective thinking processes involved: 1) critically examining both their own individual and instructional team members' ingrained curricular and instructional beliefs; and 2) gradually enlarging and evolving their consensual view of what a $21^{\text {st }}$ century elementary classroom can be. For team participants, this included arriving at a new generative metaphoric way of thinking about instructional 
planning for technology-integrated classrooms-a new way of thinking about teaching and learning as information-rich, globally-connected, and "unbounded".

Stakeholders' involvement in developing case scenes and related reflective components of the case simulation provided these school community members with a unique opportunity to critically reflect upon and develop more nuanced understandings of the web of interacting (and, often conflicting) role player beliefs and perspectives that was so central to the evolution of their current dilemma situation. Through immersing themselves in the project's creative development and refinement activities, case development team members became, in an intriguing and fundamental way, collaborative problem solving bricoleurs - “mental tinkerers” working with whatever materials and ideas they have before them (Papert, 1980: pp. 21, 76)as they critically examined each others' role perspectives and explored new avenues to inform their constructivist learning. Importantly, the case simulation development process itself provided a means for team participants to arrive at a critical organizational learning insight: that in order to be able to effectively address their school community's intractable dilemma challenges, these school community stakeholders would have to embrace the broader collaborative payoffs of learning how to work together to find a creative way-as an organizational team - to reconcile their perspectivist differences and build a practical, working consensus on "who we are and where we want to go as a collaborative teaching, leading, and learning community". In short, this group of educators and community members was challenged as collaborative stakeholder participants in their own multimedia case learning project activities to learn how to become a cohesive and critically reflective organizational learning team.

\section{Multimedia Case Development Project Activities Were Found to Be Useful as Creative Opportunities for School Community Stakeholders to Identify and Leverage Their Own Unique Organizational Core Competencies to Reinvent Themselves as Learning Communities}

One critical insight that emerged from the collective organizational case learning activities school community stakeholders engaged in during the project development year was the consensus that developed among case development participants that the "real challenge" for teachers, administrators, parents, and other community stakeholders in this school was to tap into their collective potential as an organization to learn how to think differently and work together in new ways. And, this new way of thinking and working together required these educators to recognize, more fully understand, and leverage the power of collaborative teaming-one of their newly discovered, and potentially very powerful, unique core competencies. Through engaging in "collaborative team unit planning” (multiple-silo thinking and planning) to enhance their instructional planning efforts, rather than relying exclusively on "individual lesson planning" (single-silo thinking and planning), educators in this elementary school gradually became more open to the possibilities of harnessing the internet as a collaborative team resource "tool" to energize and positively transform their collective classroom teaching and learning environments. Through leveraging collaborative team unit planning as a means to reconceptualize and revitalize how they planned together, these teachers began to find new, context-specific ways to utilize the internet and available digital learning resources to help them connect their classrooms together-to "connect the dots" for their students across lesson content, across classrooms, across grade levels, and across the street to the real world. In short, these teachers began to see the value-the "instructional payoffs"- of working together to leverage the internet and related digital learning technologies as integrative tools to more effectively enhance teaching and learning for all learners throughout the school's broader instructional environment.

Perhaps one of the most important organizational learning insights team members took away from their collective case development project experiences was the realization that, in order to be able to develop the capacity for "refashioning shared organizational identities" to move their school community forward, educators in this elementary school would need to make the critical, reflective examination of their own core beliefs about teaching and learning, the nature and purpose of learning environments, the curriculum, and the instructional units that operationalize that curriculum a central conscious part of their daily shared professional practice. In short, these educators learned that they would need to develop-as one of their key organizational core competencies-the ability as a schoolwide team to continually assess in practical ways the extent to which their individual and collective instructional beliefs are in sync with the challenges and demands of $21^{\text {st }}$ century classrooms and $21^{\text {st }}$ century learners.

\section{Stakeholder Involvement in Organizational Case Learning Holds Potential for Fostering an Emerging Culture of Distributed Leadership and a Renewed Collaborative Commitment to Positive School Change}

Stakeholders' collective experiences in this elementary school's organizational case learning project activities seemed to reinvigorate participants' collective sense of organizational efficacy and provided a new avenue for reenergizing stakeholders' commitment and capacity for positive school change. Teachers' involvement in the case development process forced these elementary teachers to confront head-on and to consciously reflect upon some of their own entrenched "attitudes and beliefs" about classroom teaching and learning-beliefs, in fact, that were holding these teachers back and preventing them from working together to develop an effective academic team mindset. Through participating in organizational case development activities, teachers were able over time to begin to fashion among themselves a new sense of shared ownership in the school's leadership challenges.

In essence, a growing collegial understanding began to evolve among stakeholder participants in the case learning project that the responsibility for active change leadership at their school did not and need not reside exclusively within one or more individual stakeholder role positions, but that this change leadership responsibility could be most usefully thought of as being distributed dynamically among all the school's stakeholders. And, it is this evolving sense of the power of distributed leadership (Harris, 2009) that enabled these school stakeholders-as an organizational case learning team - to be able to take that crucial change leadership step to begin to address directly their own "crisis of instructional and organizational 
leadership confidence" and, in doing so, begin to foster in their school community an emerging culture of shared leadership responsibility for positive school change.

The organizational case development strategies that were utilized in this school-university collaborative initiative as the basis for assisting these elementary school stakeholders in building a revitalized culture of shared leadership responsibility within their school community were firmly grounded in a phenomenological approach to human learning (Van Manen, 1990). As a form of qualitative inquiry, phenomenological analysis emphasizes the relevance and importance to individuals and groups of reflecting critically on their own lived experiencestheir real-life perceptions and perspectives-as a central starting-off point for evolving insightful understandings about and making sense of those experiences (Smith, et al., 2009). The multimedia case development project activities engaged in by these elementary school case team participants and reported here were designed specifically as a structured means to enable these school stakeholders to immerse themselves in a unique and highly context-specific organizational team-learning endeavor. A central focus of this endeavor was on engaging participants in working together in a new way as a collaborative case development team to critically analyze and reflect on their collective interactive experiences within the context of their own real-world school leadership challenges. Coming together in this manner as a case development team, school stakeholder participants were provided with a new means and a unique vantage point for engaging in focused critical, reflective analyses of their overall school community interactive experiences. School stakeholders' collective case development and analysis project efforts represented for participants an alternative team learning platform for gaining new shared understandings about important school community issues and challenges, and an opportunity to interpret and make sense of their shared reality in new, creative ways (Weick, 1995).

The advantages of developing and nurturing an organizational team reflective mindset as a means for energizing and revitalizing a positive culture of distributed leadership within a school community were discussed openly by university and school case team participants both during and following the completion of case development project work at this elementary school. One teacher's comments at the conclusion of project activities summarized the renewed sense of positive change leadership capacity that developed and was felt among case team participants: "We realize that no single initiative, in and of itself, can solve our school improvement challenges. But this project has forced us to think differently about ourselves and to reevaluate how we might work together to better understand who we are as a school community and how to effectively address our challenges. We already knew what many of our school improvement challenges were and the general overall direction we needed to be moving in, but, as members of the same school community, I think we now have a better sense of the kind of common school culture we need to build together, and how important that culture will be in helping us be able to achieve our school improvement goals.”

Of course, nurturing an energized culture of distributed leadership within any school leading and learning community is an organizational challenge that requires sustained effort over time. Thus, using multimedia case design and development as an alternative, immersive organizational learning tool may be best employed as one creative component of a larger, integrated set of short- and long-term organizational learning and development strategies to help build and maintain among school community stakeholders a revitalized sense of shared organizational purpose and a lasting commitment to positive school improvement.

\section{Conclusion}

This article has reported on activities and results associated with a multimedia case learning and development project involving educators and school community stakeholders in one West Texas elementary school. The project's design utilized concepts and techniques associated with dramatic theatre, cinematography, and collaborative staff development to involve stakeholder participants and project personnel in a unique organizational case learning and development experience using available multimedia technology.

A key feature of the case development process described herein was the leveraging of school stakeholders' own lived experiences and school community challenges as the contextspecific basis for immersing stakeholders in an alternative staff development and organizational team learning adventure that forced stakeholder participants to examine critically and reflectively their real-world dilemma situation in new, multi-perspectivist ways. Project activities enabled school community stakeholders to come together in a new way to examine and explore their own real-world school dilemma challenges as a creative multimedia case development team. As a result of their involvement in project activities, stakeholder participants at this elementary school were able to glean new real-world insights on how to transform themselves into effective organizational leading and learning "inceptors"-that is, to develop the capability to critically and reflectively refashion their communal "idea" of themselves as collaborative and transformative school leaders. In doing so, these school community stakeholders were able to reshape and revitalize their owned shared sense of organizational identity to build the collaborative leadership capacity to help them move their school community forward. Most importantly, all of us involved in this school-university partnership endeavor (both university research and school community teams alike) gained valuable new understandings and respect for the potential of multimedia technology as a useful tag-teaming tool for helping stakeholders in organizations think differently about leadership and learn how to work together more effectively in new ways.

\section{References}

Harris, A. (2009). Distributed leadership: Different perspectives. London: Springer.

Nolan, C. (2010). Inception: The shooting script. San Rafael, CA: Insight Editions.

Papert, S. (1980). Mindstorms: Children, computers, and powerful ideas. New York: Basic Books.

Prensky, M. (2010). Teaching digital natives: Partnering for real learning. Thousand Oaks, CA: Corwin.

Smith, J.A., Flowers, P., \& Larkin, M. (2009). Interpretative phenomenological analysis. London: Sage Publications.

Van Manen, M. (1990). Researching lived experience. Albany, NY: 
State University of New York Press.

Weick, K. E. (1995). Sensemaking in organizations. Thousand Oaks, CA: Sage Publications.

Weisbord, M. (1992). Discovering common ground: How future search conferences bring people together to achieve breakthrough innova- tion, empowerment, shared vision, and collaborative action. San Francisco, CA: Berrett-Koehler Publishers.

Wheatley, M. J. (1999). Leadership and the new science: Discovering order in a chaotic world (2nd ed.). San Francisco: Berrett-Koehler Publishers. 